\title{
PREFACE
}

\section{Second Thematic Meeting of CRAYNET, Halden Norway, 1-4 September 2003}

This volume is the second in a series of Proceedings of the various meetings of the European Thematic Network CRAYNET "European crayfish as keystone species - linking science, management and economics with sustainable environmental quality".

The CRAYNET European contract was signed in November 2002 and followed by a planning meeting in Paris in January 2003, attended by the core members. Dates were agreed for the three thematic meetings, each concentrating on a particular topic relevant to one of the native European species. The first thematic meeting, on the endangered white-clawed crayfish Austropotamobius pallipes as bioindicator and heritage species, was held in Kilkenny, Ireland, 22-24 June 2003. (Bull. Fr. Pêche Piscic., 370-371.)

The second thematic meeting, of which the outcome is presented in this volume, was entitled "European native crayfish with a focus on Astacus astacus: linking socioeconomics and conservation". The small city of Halden in Southeastern Norway, was selected as conference site. Halden is located in the heart of the Norwegian crayfish district along one of the most tradition-rich crayfish catching watercourses and also close to Sweden, the country from which Norwegians have learnt to love the crayfish. The special objective of the Halden meeting was to focus on the cultural and socioeconomic significance of the noble crayfish, Astacus astacus, and the important link between conservation and use.

As an indicator of the interest in crayfish in Sweden the number of articles each week containing the words signal crayfish, noble crayfish, crayfish or crayfish plague is shown below. During the start of the crayfish season in August the number of articles exceeds 100 a week. An alternative way of showing how important the crayfish are is by comparing the size of supplements that are put in the main evening newspapers. The supplement distributed during the European football championship in Portugal 2004 contained 34 pages. The one dealing with crayfish included in the newspapers at the start of the crayfish season in August 2004 contained 68 pages!

The program of the second CRAYNET meeting comprised plenary talks, poster presentations and roundtable discussions. A total of 14 plenary talks addressed sociocultural and socioeconomic aspects, conservation and restocking, trade regulations, genetic variation, survey methods, and perspectives on the past, present and future of crayfish pathology in Europe. One presentation which is also chosen as an introductory paper of this volume, was given by Prof. Jan-Öjvind Swahn from the Dept. of European Ethnology of the Lund University in Sweden. He focused on the cultural history of crayfish and the important role the crayfish has played in European eating habits, habits that in many countries have become extinct. In the Scandinavian countries the present ritual and ceremonial form of social intercourse, called the "The Crayfish Party", developed during the second half of the nineteenth century and has been an extremely popular latesummer tradition ever since. The sociocultural aspect is very important to bear in mind when discussing and implementing management and conservation strategies. The poster sessions included 17 posters covering a wide range of topics and crayfish species. The roundtable discussions included four parallel sessions on the following topics: 1) Threats to native crayfish populations - crayfish on a landscape level, 2) Exploitation, conservation and legislation, 3) Reintroduction of native crayfish, habitat restoration and monitoring, and 4) Crayfish diseases. The roundtable session finished with a plenary presentation of conclusions and recommendations from the different groups. 


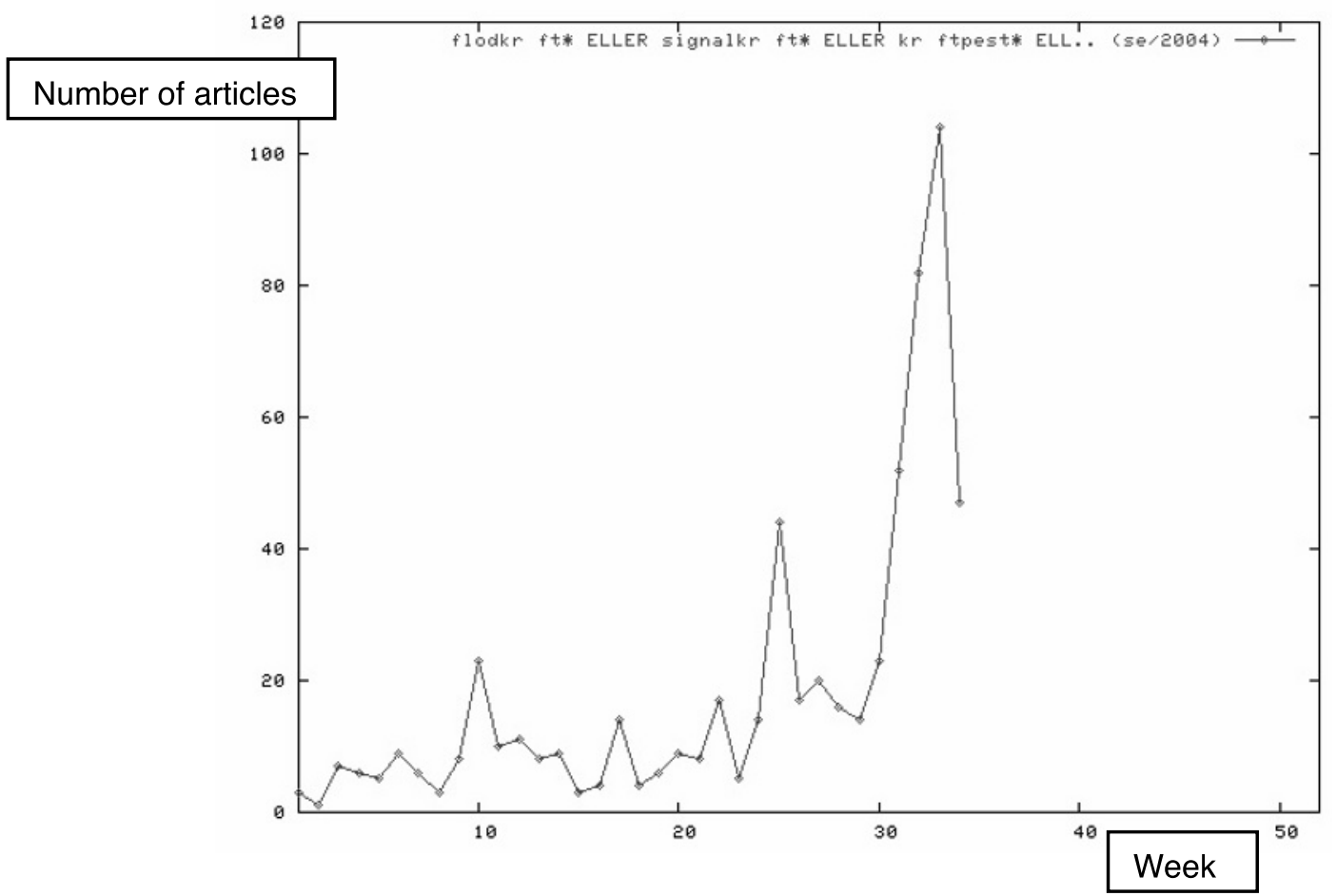

Articles dealing with crayfish issues published in Swedish newspapers each week between January $1^{\text {st }}$ and September $5^{\text {th }} 2004$.

The meeting ended with a traditional crayfish party, Scandinavian style. The event took place in idyllic surroundings at the shore of Lake Rødenessjøen. A total of $45 \mathrm{~kg}$ of noble crayfish was consumed together with necessary accessories. As usual at crayfish parties, the participants got inspiration for singing and dancing. The aim of the crayfish party was not only social, but also to demonstrate the magic of crayfish eating in a social assembly, and give some impressions of the cultural significance of crayfish in Scandinavia.

The CRAYNET-meetings aim to bring together not only the core scientists but also other crayfish researchers and managers, to identify necessary research for a common approach to management techniques, and to develop recommendations for optimal management strategies at a European scale. The Halden CRAYNET meeting gathered 58 participants from 17 countries. The CRAYNET-meeting was immediately followed by a national Swedish/Norwegian meeting on the same topic gathering some 80 participants from both countries. This meeting was organised by the Swedish-Norwegian EU-Interreg project on crayfish conservation and use in collaboration with CRAYNET. The close link between these meetings was aimed at bridging the gap between the international level/ scientific research and local managers and stakeholders.

The ongoing emphasis within CRAYNET is to promote and develop knowledgebased management and public awareness of European crayfish. Our conclusions, that species complexes are imperfectly served under current legislative structures and that controlled fishing may not be incompatible with exploitation, are already understood by many anglers and naturalists, but have yet to receive wider management recognition. Crayfish play an important role as controllers of biodiversity as well as representing 
heritage value biodiversity in their own right. Education is of paramount importance, and so we are concentrating on this through our website, through a discussion forum reaching some 500 correspondents, and through the preparation of educational materials.

Guidelines for managers and other stakeholders are in preparation, as is an Atlas of crayfish distribution in Europe, to be completed under the auspices of the Museum National d'Histoire Naturelle in Paris. To this end we have started to create a large biogeographical database, which will increase in value and usefulness with time. If the biodiversity implications of Gothenburg are to be realised by 2010, a database such as this should not end with the publication of the atlas in 2005.

Trond TAUGBøL, Organiser of the Halden thematic meeting, Norway Lennart EDSMAN, National Board of Fisheries, Sweden Catherine SOUTY-GROSSET, Co-Ordinator of Craynet, France 



\section{PRÉFACE}

\section{Second Thematic Meeting of CRAYNET, Halden, Norvege, 1-4 Septembre 2003}

Ce volume est le premier d'une série relatant les travaux effectués au cours des réunions organisées au sein du réseau thématique européen CRAYNET «European crayfish as keystone species - linking science, management and economics with sustainable environmental quality ».

Le réseau européen CRAYNET a été signé en novembre 2002 et fut suivi par une réunion de démarrage du projet à Paris, avec tous les pays membres, en janvier 2003. Les dates concernant trois réunions thématiques furent arrêtées, chacune étant concentrée sur un thème particulier et consacrée à l'une des espèces natives d'Europe. La première réunion thématique sur l'écrevisse à pattes blanches Austropotamobius pallipes en tant que bioindicateur et espèce patrimoniale menacée eut lieu à Kilkenny, Irlande, 22-24 Juin 2003. (Bull. Fr. Pêche Piscic., 370-371.)

La seconde réunion thématique, faisant l'objet du présent volume, est intitulée "European native crayfish with a focus on Astacus astacus: linking socioeconomics and conservation ". La petite cité d'Halden située dans le sud-est de la Norvège a été sélectionnée comme site de rencontre. Halden est située au coeur d'un district norvégien possédant des écrevisses, proche des ruisseaux les plus traditionnellement riches en écrevisses et également proches de la Suède, pays ayant appris aux norvégiens à apprécier les écrevisses. L'objectif particulier de la réunion d'Halden était de se concentrer sur la signification culturelle et socioéconomique de l'écrevisse à pattes rouges (dite écrevisse noble) Astacus astacus, et sur le problème important du lien entre conservation et consommation.

Pour illustrer l'intérêt pour les écrevisses en Suéde, il suffit d'examiner ci dessous le nombre d'articles parus chaque semaine et comprenant les mots écrevisse signal, écrevisse noble ou peste des écrevisses. Au début de la saison des écrevisses en août, le nombre d'articles dépasse 100 par semaine. Une autre façon de montrer comme les écrevisses sont importantes est de comparer la taille des suppléments parus dans les principaux journaux du soir. Le supplément distribué durant le championnat de football d'Europe au Portugal en 2004 faisait 34 pages. Celui ayant trait aux écrevisses et paru au début de la saison estivale des écrevisses en août 2004 faisait 68 pages!

Le programme de la deuxième réunion de CRAYNET a compris des communications orales, des présentations de poster et des discussions au sein de tables rondes. 14 communications orales ont concerné les aspects socioculturels et socioéconomiques, la conservation et le repeuplement, la législation et le commerce, la variabilité génétique, les méthodes d'inventaire, et les perspectives sur le passé, le présent et le futur concernant la pathologie des écrevisses en Europe. La présentation orale, qui a été choisie pour introduire ce volume, a été faite par le Professeur. Jan-Öjvind Swahn du Département d'Ethnologie Européenne de l'Université de Lund en Suéde. II s'est attaché à retracer l'histoire culturelle des écrevisses et le rôle important qu'elles ont joué dans les coutumes culinaires en Europe, coutumes actuellement disparues dans beaucoup de pays. Par contre, dans les pays scandinaves la forme actuelle rituelle et cérémoniale en société, appelée "The Crayfish Party ", s'est développée au cours de la seconde moitié du dix neuvième siècle et, depuis, est une tradition extêmement populaire en fin d'été. L'aspect socioculturel est très important à prendre en compte quand il s'agit de discuter et de mettre au point des stratégies de gestion et de conservation. Les sessions poster 


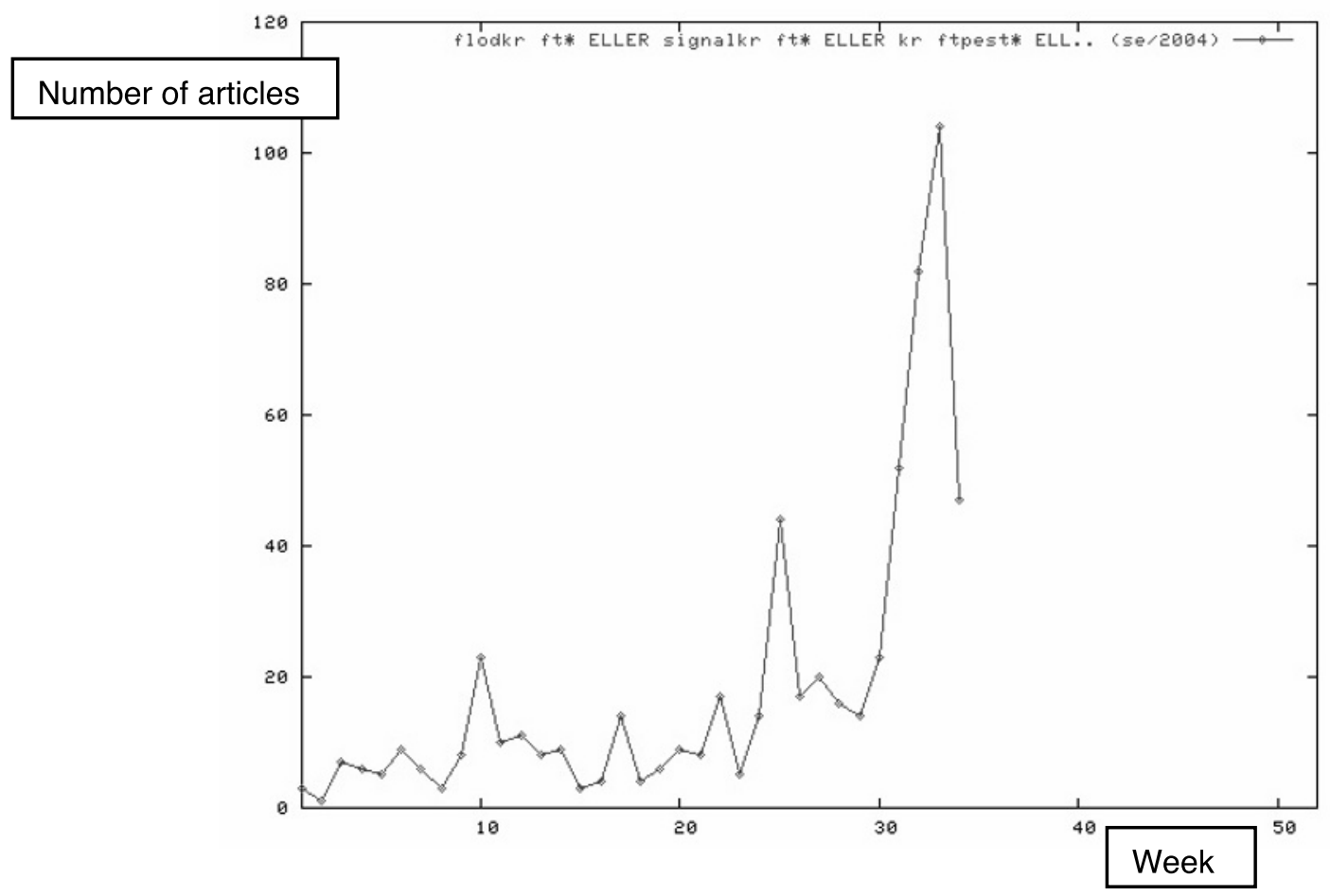

Articles ayant trait aux écrevisses et parus dans des journaux suédois chaque semaine entre le $1^{\text {er }}$ janvier et le 5 septembre 2004.

ont compris 17 posters couvrant une grande variété de thèmes et d'espèces d'écrevisses. Les discussions au sein des tables rondes ont inclus quatre sessions parallèles sur les thèmes suivants : 1) Menaces pour les populations d'écrevisses - écrevisses et paysages, 2) Exploitation, conservation et législation, 3) Réintroduction des écrevisses natives, restauration de l'habitat et suivi, ainsi que 4) Pathologie des écrevisses. La session des tables rondes a donné suite à une séance plénière pour présenter les conclusions et les recommandations des différents groupes de travail.

Le programme final de ce meeting a inclus une traditionnelle "crayfish party ", selon le style scandinave. La fête a eu lieu dans le cadre idyllique des berges du lac Rødenessjøen. Un total de $45 \mathrm{~kg}$ d'écrevisse noble a été consommé par les congressistes avec le matériel festif nécessaire. Comme c'est la tradition dans ces "crayfish parties ", les participants ont trouvé l'inspiration pour chanter et danser. Le but de cette " crayfish party " était non seulement d'ordre social, mais aussi de démontrer l'aspect magique de partager un plateau d'écrevisses dans une ambiance festive et conviviale, et de faire appréhender les impressions de la signification culturelle des écrevisses en Scandinavie.

Les réunions CRAYNET ont pour but de rassembler non seulement les scientifiques membres du réseau mais tous les scientifiques européens et les gestionnaires, afin d'identifier la recherche à entreprendre pour une approche commune des techniques de gestion et de développer des recommandations pour une stratégie de gestion optimale à l'échelle européenne. La réunion CRAYNET d'Halden a rassemblé 58 participants de 17 pays. Cette réunion CRAYNET a été immédiatement suivie par un congrés national Suède/Norvège sur le même thème et rassemblant 80 participants des deux pays. Ce meeting a été organisé par le Swedish-Norwegian EU-Interreg projet sur la conservation des écrevisses et usage en collaboration avec CRAYNET. Le lien étroit entre ces deux 
meetings était d'établir le pont entre la recherche scientifique au niveau international et les gestionnaires et tous les acteurs partie prenante.

Le travail continuel de CRAYNET est de promouvoir et de développer la connaissance et la gestion ainsi que l'éducation du public au sujet des écrevisses natives d'Europe. Les conclusions que les complexes d'espèces sont mal protégés sous la législation actuelle et que la pêche contrôlée ne doit pas être incompatible avec l'exploitation sont déjà comprises par beaucoup de pêcheurs et de naturalistes mais doivent être plus largement reconnues en terme de gestion. Les écrevisses jouent un rôle important en tant qu'espèces contrôlant la biodiversité et également comme espèces à valeur patrimoniale reconnue. L'éducation est de la plus haute importance et nous insistons particulièrement sur cet aspect sur notre site web, au travers d'un forum électronique auquel près de 500 correspondants sont abonnés, et par la préparation de brochures éducatives.

Des guides pour les gestionnaires et tous les acteurs partie prenante sont en préparation ainsi qu'un Atlas de distribution des écrevisses en Europe, avec le concours du Museum National d'Histoire Naturelle de Paris. Dans ce but nous avons commencé par créer une grande base de données biogéographiques qui augmentera en valeur et en utilité avec le temps. Si des retombées en termes de biodiversité (Gothenburg) doivent être sensibles pour 2010, une telle base de données ne devrait pas être arrétée avec la publication de l'atlas prévue en 2005.

Trond TAUGBøL, Organiser of the Halden thematic meeting, Norvège Lennart EDSMAN, National Board of Fisheries, Suède Catherine SOUTY-GROSSET, Co-Ordinator of CRAYNET, France 
